\title{
A Systematic Review Study on Self-Care in Diabetic Patients: The Most Important Factors Affecting the Self-Care of These Patients Malihe Salimi ${ }^{1}$, Mahboobe Khodadadi ${ }^{2}$, Samaneh Naeimi ${ }^{3 *}$
}

1B.Sc, Nursing student in M.Sc, Department of Nursing, School of Nursing and Midwifery, Ahvaz Jundishapur University of Medical Sciences, Ahvaz, IR Iran.

${ }^{2}$ M.Sc, student of nursing, Student Research Committee, Ahvaz Jundishapur University of Medical Sciences, Ahvaz, Iran.

${ }^{3}$ Nursing Student, Student Research Committee, Abadan Faculty of Medical Sciences, Abadan, Iran.

\section{ABSTRACT}

Introduction: Diabetic patients are aware of the principles of proper nutrition, choice of foods, careful observation of treatment pattern, foot care, exercise and appropriate physical activity along with lifestyle changes and mental and health care. They play a role in preventing short-term complications. Observing these will also delay the progression of long-term complications of diabetes. Achieving such goals undoubtedly requires patient participation in self-care and dynamic and continuous education, so that without self-care training for diabetic patients and their participation in self-care, more expensive health care, and impaired quality of life. Therefore, this systematic review study was done on most important factors affecting of self-care in diabetic patients.

Materials and Methods: In this systematic review, in order to achieve the goal of the study and to improve the study's precision and its comprehensive understanding, this integrated overview study was conducted based on the Broome method. Broome's method is based on three stages of the search of texts, data evaluation and data analysis, so that in the search phase, the texts of postretrospective studies are examined in terms of the criteria for entering the study in four stages and after obtaining entry conditions The content of the study is evaluated and the data is analyzed at the end.In the first stage, 43 papers were found. Of these, 12 articles related to the topic that were published in the last 23 years were reviewed.

Results: In this article, 12 studies were reviewed. In one of these studies, there was a significant relationship between self-care and patient visit in the clinic that Knowledge and education in the referring subjects increased their mean self-care score

Conclusion: According to the results of the studies, diabetic patients have poor self-care, and this poor self-care imposes huge costs on affected individuals each year. Therefore, it is necessary to find ways to improve the care of these patients.

Keywords: Diabetic Patients, Diabetes, Self-Care, Most Important Factors

\section{Address for Correspondence Author}

Samaneh Naeimi, Nursing Student, Student Research Committee, Abadan Faculty of Medical Sciences, Abadan, Iran. E-mail: samanehnaeimi1377@gmail.com

\section{Introduction}

Until recently, contagious diseases were world countries. But now the increasing role of considered the biggest health problem in third non-communicable diseases in mortality, 
especially in developing countries, is a serious threat, one of which is diabetes. Diabetes is one of the most important global challenges as the most common disease caused by metabolic disorders. It is a chronic, metabolic disease, and is clinically and genetically heterogeneous. And a major cause of morbidity and mortality in the industrialized and developing world (1-7).

At present, the prevalence of type 2 diabetes in Iran is $7.7 \%$. People with diabetes with a body mass index (BMI) above a "normal" classification are at increased risk of secondary complications of diabetes (8-11).

Complications of diabetes are extremely common among patients, including ocular, renal, peripheral neuropathy, and peripheral arterial disease (12-16).

Because of its chronic nature, diabetes has affected the quality of life of these patients. Complications of this disease impose heavy economic burden and decrease the quality of life of these patients $(17,18)$.

Diabetic patients are aware of the principles of proper nutrition, choice of foods, careful observation of treatment pattern, foot care, exercise and appropriate physical activity along with lifestyle changes and mental and health care. They play a role in preventing short-term complications. Observing these will also delay the progression of long-term complications of diabetes. Achieving such goals undoubtedly requires patient participation in self-care and dynamic and continuous education, so that without self-care training for diabetic patients and their participation in self-care, more expensive health care, and is impaired quality of life. There will be more. Implementing a self-care program will improve the care and quality of life of diabetic patients $(19,20)$.
Therefore, this systematic review study was done on most important factors affecting of self-care in diabetic patients.

\section{Materials and Methods}

Criteria for entry were articles, including published articles in Persian and English, full access to their full text, published over the past 23 years, and interventional studies that focused on the impact of peer education on patient self-care behaviors. Exclusion criteria include unspecified and unscientific studies as well as articles that do not have the full text of the article.

In this systematic review, in order to achieve the goal of the study and to improve the study's precision and its comprehensive understanding, this integrated overview study was conducted based on the Broome method. Broome's method is based on three stages of the search of texts, data evaluation and data analysis, so that in the search phase, the texts of post-retrospective studies are examined in terms of the criteria for entering the study in four stages and after obtaining entry conditions The content of the study is evaluated and the data is analyzed at the end.

This study uses the published articles in the last 23 years on the effects of some factors on self-care in diabetic patients. It was conducted in English and Persian by searching articles in search engines, authoritative scientific sites and databases Google Scholar, PubMed, Springer. In the first stage, 43 papers were found. Of these, 12 articles related to the topic that were published in the last 23 years were reviewed.

To achieve relevant studies, a wide range of keywords including Diabetic patients, Diabetes, Self-care and most important factors was used as a one-to-one search, combined with the method "And" and "OR". 


\section{Results}

Diabetic patients are aware of the principles of proper nutrition, choice of foods, careful observation of treatment pattern, foot care, exercise and appropriate physical activity along with lifestyle changes and mental and health care. Observing these will also delay the progression of long-term complications of diabetes. Achieving such goals undoubtedly requires patient participation in self-care and dynamic and continuous education, so that without self-care training for diabetic patients and their participation in self-care, more expensive health care, and is impaired quality of life. There will be more. Implementing a self-care program will improve the care and quality of life of diabetic patients $(19,20)$.

In some studies, comparing the mean scores of behaviors in different aspects of self-care showed that the mean behaviors in terms of diet and proper use of medications had the highest and least effect on blood glucose control and physical activity (21-28).

A study of trained individuals by untrained physicians and health personnel found that the mean score of self-care in trained individuals was 3.66 points higher than those who did not receive training (22).

Also, in some studys, there was a significant relationship between self-care and patient referral in the clinic, which increased the level of knowledge and education in clients, increasing the mean score of self-care $(22,27)$.

Also, self-care status was significantly correlated with the duration of education, so that self-care increased as self-care increased and illiterate people had good self-care. According to the results of this study, the type of treatment and marital status relates only to foot care $(29,30)$.
Also, the results of some studies showed that $47.2 \%$ of the subjects had a poor self-care and there was a significant relationship between selfcare and sex and physical activity (31).

In diabetic patients, self-care education is one of the key approaches to successful diabetes management. Teach Back is an interactive educational tool that can enhance knowledge and understanding of the patient and improve their self-management.

In a study (32), that was done with aims determining the effect of Self-care education through Teach Back method on self-care behaviors in type 2 diabetic patients, showed that after the intervention, the patients in the experimental group had a good performance and control group had a moderate performance on blood glucose control.

According to the results of this study, after the intervention, the mean score of awareness of the case group in comparison with the control group increased significantly. This is in line with the level of awareness of type 2 diabetic patients about self-care in the study of neonates and cohorts (33).

\section{Discussion}

Until recently, contagious diseases were considered the biggest health problem in third world countries. But now the increasing role of non-communicable diseases in mortality, especially in developing countries, is a serious threat, one of which is diabetes. Diabetes is one of the most important global challenges as the most common disease caused by metabolic disorders. It is a chronic, metabolic disease, and is clinically and genetically heterogeneous. And a major cause of morbidity and mortality in the industrialized and developing world (1-7). 
The importance and the crucial role that self-care has in controlling, promoting the health and quality of life of diabetic patients, and preventing the development of complications and disabilities, is well known (33), Therefore, this systematic review study was done on most important factors affecting of self-care in diabetic patients.

In a study (33), before education, there was no significant difference between the case and control groups regarding the knowledge of diabetic patients about self-care. But patients' awareness after training was significantly increased in both case and control groups, which was higher and more significant in case group. Also in the case group, there was a significant difference after regular training compared to before, but no significant difference was observed in the control group before and after training. Exercise in the case group increased significantly after training compared to the previous group. But in the control group, no significant difference was observed before and after the intervention.

Diabetes self-care may be a function of the individual, psychological, and social factors that understand health care providers to develop effective interventions to promote diabetes selfcare behaviors (28)

According to study (29), Based on the results of this study, the type of treatment and marital status were related only to the foot care dimension, it can be said that due to the results of the study and the differences in some characteristics of the patient, patients with different dimensions of self-care and knowledge need training in diabetes centers more practical.

\section{Conclusion}

According to the results of the studies, diabetic patients have poor self-care, and this poor selfcare imposes huge costs on affected individuals each year. Therefore, it is necessary to find ways to improve the care of these patients.

\section{Acknowledgment}

The author of this article is grateful to all those who have collaborated with me in this article.

\section{References}

1. Khanpaye A, Abdoullahi A, Riahipour B, Madmoli Y, Mohebifar M, Mahmoodi R, Shirmardi S, Madmoli M. Evaluation of Knowledge, Attitude and Performance Regarding Gestational Diabetes Mellitus in Southwest of Iran. Journal of Pharmaceutical Research International. 2019 Jul 11:1-6.

2. Moslemirad M, Madmoli M, Madmoli Y, Niksefat M. Prevalence of type 1 and type 2 diabetes and its related factors in diabetic patients hospitalized in Khatamol-Anbia hospital in Shoushtar, 2014-15: A retrospective study. Journal of Research in Medical and Dental Science. 2018;6(3):421-6

3. Feyzi R, Madmoli M. Epidemiologic approach to quality of life in diabetic patients: a systematic review study. IJMBS [Internet].2019;3(5): 24-28. Available from:

http://www.ijmbs.info/index.php/ijmbs/ article/view/226

4. Madmoli M, Madmoli M, Aliabad MA, Khodadadi M, Ahmadi FP. A systematic review on the impact of empowerment in improving self-care behaviors and some other factors in diabetic patients. International Journal of Health and Biological Sciences. 2019 Apr 13;2(1):116.

5. Rostami F, Madmoli M, MirsamiYazdi N, Baraz Sh. Evaluation of The Prevalence of Lower Limb Amputation and Its Related Factors in Diabetic Patients Admitted to 
KHatam-ol-Anbia Hospital in Shoushtar During The 2015-2016: A Retrospective Study. International Journal of Ecosystems and Ecology Science (IJEES). 2018 June; 8 (3): 553-60.

6. Madmoli $M$, Izadi $M$, Khodadadi $M$, Khodadadi M, Papi Ahmadi F, Abbaszade Aliabad M.A Systematic Review Study on the Effect of Multimedia Training on SelfCare Behaviors and Quality of Life in Diabetic Patients. Journal of Genetics and Genetic Engineering, 2019; 2(4): 1-6.

7. Raisifar Z, Afshar Nia A, MaghamesiMoarrefi H, Madmoli M. Evaluation of Gi Bleeding Prevalence and Its Related Factors in Diabetic Patients Hospitalized in KHatam-ol-Anbia Hospital During 2015-16: A Retrospective Study. International Journal of Ecosystems and Ecology Science (IJEES). 2018 June; 8 (3): 609-14.

8. Madmoli M, Modheji Y, Rafi A, Feyzi R, Darabiyan P, AfsharNia A. Diabetes and its predictive role in the incidence of Alzheimer's disease. Medical Science, 2019; 23(95), 30-34

9. Madmoli M Rostami F, MirsamiYazdi N, Mosavi A, Baraz Sh. Evaluation of Prevalence of Diabetic Foot Ulcer and Its Related Factors in Diabetic Patients Admitted to KHatam-ol-Anbia Hospital in Shoushtar During 2015-2016: A Retrospective Study. International Journal of Ecosystems and Ecology Science (IJEES). 2018 June; 8 (3): 545-52.

10. Madmoli M, Madmoli Y, Taqvaeinasab H, Khodadadi M, Darabiyan P, Rafi A. Some influential factors on severity of diabetic foot ulcers and Predisposing of limb amputation: A 7-year study on diabetic patients. International Journal of
Ayurvedic Medicine. 2019 Apr 3;10(1):7581.

11. Madmoli M, Abbaszade Aliabad M, Madmoli M, Khodadadi M, Papi Ahmadi F. The Effect of Some Factors on Self-Care in Diabetic Patients: A Systematic Review. Journal of Genetics and Genetic Engineering. 2019; 3(1): 21-25

12. Madmoli M. A systematic Review Study on the Results of Empowerment-Based Interventions in Diabetic Patients. Int. Res. Med. Health Sci., 2019; 2(1):1-7.

13. Madmoli M, MahmoudiDehcheshmeh Z, Rafi A, Zahra Kord, FaribaMobarez, Pouriya Darabiyan. The rate of some complications and risk factors of diabetes in diabetic patients: Study on cases of 3218 diabetic patients. Medical Science, 2019; 23(95), 63-68

14. Madmoli M, Madmoli Y, Khodadadi M, Samsamipour M. Study of Some Effective Treatments for Accelerating Diabetic Foot Ulcer Healing: A Systematic Review. International Journal of Research Studies in Science, Engineering and Technology. 6(2), 2019; 34-39

15. Madmoli M, Madmoli Y, Khodadadi M, Samsamipour M. Factors Affecting the Level of Glycosylated Hemoglobin in Patients with Diabetes: A Systematic Review. Annals of Microbiology and Infectious Diseases 2(1), 2019; 43-47.

16. Madmoli M, Madmoli Y, Khodadadi M, Samsamipour M. Some Factors Affecting Quality of Life in Patients with Diabetes: A systematic Review. Annals of Microbiology and Infectious Diseases, 2(1), 2019; 26-30.

17. Adachi $M$, Yamaoka $K$, Watanabe $M$, Nishikawa M, Hida E, Kobayashi I, et al. Effects oflifestyle education program for type 2 diabetes patients in clinics: Study 
design of a clusterrandomized trial. BMC Public Health. 2010; 10: 742.

18. Raaijmakers LG, Hamers FJ, Martens MK, Bagchus C, De Vries NK, Kremers SP. Perceivedfacilitators and barriers in diabetes care: A qualitative study among health care professionals inthe Netherlands. BMC Family Practice. 2013; 14: 114.

19. Bate KL, Jerums G. Preventing complications ofdiabetes. Med J Aust 2003; 179(9):498-503.

20. Vargas-Lombardo M, Jipsion A, Vejarano R, Camargo I, Álvarez H, Mora EV, Ruíz EM. Implementation a holistic model, interactive and persuasive to facilitate self-care of patients with diabetes (hIPAPD). In2010 Second International Conference on eHealth, Telemedicine, and Social Medicine 2010 Feb 10; 57-63.

21. Rahimian BI, Mohajeri TM, Besharat M, Talepasand S. Psychological, social and structural determinants of diabetes selfmanagement. 2012; 467-478.

22. Rahimian BI, Mohajeri TM, Besharat M, Talepasand S. Psychological, social and structural determinants of diabetes selfmanagement.2012; 467-478.

23. Taghipour A, Moshki M, Mirzaei N. Determination of Effective Factors on Selfcare Behaviors in Women with Diabetes Referring to Mashhad Health Centers. Iranian Journal of Health Education and Health Promotion. 2017 Nov 15;5(4):32835.

24. Nouhjah S. Self-care behaviors and related factors in women with type 2 diabetes. Iranian Journal of Endocrinology and Metabolism. 2015 Mar 15;16(6):393-401.

25. Mazloomi S, Mody M, Malakimoghadam $\mathrm{H}$, Tavasoli E, Vahdani Nia Z. PREDICTORS OF SELF-CARE IN TYPE 2 DIABETIC
PATIENTS IN BIRJAND AND YAZD CITY. Iranian Journal of Diabetes and Metabolism. 2018 Mar 15;17(2):97-104.

26. Abbaspour S, Shamaeiyan N, Hasanzadeh M, Zandi Z, Sepehri A. Self-care behaviors among diabetic patients referred to a selected hospital clinic in TorbateHeydarieh. Journal of TorbatHeydariyeh University of Medical Sciences. 2013;1(1):65-70.

27. Shakibazade E, Larijani B, Rashidian A, Shojaazadeh D, Forouzanfar $M \quad H$. Comparison of Views of Patients with Type 2 Diabetes with Specialized Diabetes Specialists on Self-Care Effective Factors: Qualitative Study. Journal of Diabetes and Metabolism of Iran. 1389; 9 (2): 142-151

28. Diadarloo D, Eftekhard A, NiknamamiSh, Hajizadeh A. Factors Affecting Self-Care Behavior in Diabetic Women Referring to Diabetes Clinic in Khoy City are developed based on the theory of rational action. Quarterly Journal of School of Public Health and Institute of Health Research. Nov. 15, 2011; 9 (2): 79-92.

29. Baghaei P, Zandi M, Vares Z, MasoudiAlavi N, Adib-Hajbaghery M. Self-care situation in diabetic patients referring to Kashan Diabetes Center, in 2005. Feyz. 2008; 12 (1) :88-93

30. MorrowatiSharifabad M A, Rouhani Tonkaboni N. Diabetes self-care determinant pattern in patients referring to Yazd Diabetes Research Center.2013;33-38

31. Firooz, M., Hosseini, S., Mazlom, S., Hasan zadeh, F., Kimiyaee, S. Self-care of patient with diabetes type II. Journal of Sabzevar University of Medical Sciences, 2016; 22(6): 1018-1025.

32. Oshvandi K, Jokar M, Khatiban M, Keyani J, Yousefzadeh M R, Sultanian A R. The effect 
of self-care education based on teach back method on promotion of self-care behaviors in type ii diabetic patients: a clinical trial study. ijdld. 2014; 13 (2) :131-143
33. Naghibi S A, Asghari M, Rostami F. Investigation the Effect of Education on Self-Care Promotion in Type 2 Diabetic Patients in Noor Health Centers in 2015. J Health Res Commun. 2015; 1 (2) :22-28

How to cite this Article: Samaneh Naeimi*, A Systematic Review Study on Self-Care in Diabetic Patients: The Most Important Factors Affecting the Self-Care of These Patients

Int. Res. Med. Health Sci., 2019; (2-6): 5-11

Source of Support: Nil, Conflict of Interest: None declared.

Received: 08-12-2019; Revision: 21-12-2019; Accepted: 24-12-2019 